\title{
Definitions, Epidemiology and Pathophysiology
}

\author{
Stephen M. Robert ${ }^{1}$, E. Scott Halstead ${ }^{2}$, Joseph A. Carcillo ${ }^{2}$ and Rajesh K. Aneja*² \\ ${ }^{I}$ Division of Pediatric Critical Care Medicine, Children's Hospital of Alabama; Birmingham, AL, UK \\ ${ }^{2}$ Departments of Critical Care Medicine and Pediatrics, University of Pittsburgh School of Medicine and Children's \\ Hospital of Pittsburgh; Pittsburgh, PA, USA
}

\begin{abstract}
Despite significant progress in the understanding and treatment of sepsis, it continues to be a major health problem in United States and around the world. Sepsis accounted for nearly 4,500 deaths (mortality rate 10\%) and approximately \$2 billion per year in healthcare expenditures in the U.S. alone. In this review, we will revisit the definitions of pediatric sepsis and discuss the epidemiology of sepsis in the United States. During inflammatory states, the vascular endothelium plays a crucial role in modulating the host immune response and regulates the levels of coagulation, pro-inflammatory and anti-inflammatory factors in the blood. We review the role of endothelium in nitric oxide generation and activation of the coagulation cascade. Studies in critically ill patients and animal models have shown that increased apoptosis or "programmed cell death" of lymphoid organs contributes to immune suppression, anergy, and organ system dysfunction. In this chapter, we outline both intrinsic and extrinsic pathways that lead to apoptosis.
\end{abstract}

Keywords: Pediatric sepsis, septic shock, pathophysiology of sepsis.

\section{DEFINITIONS}

The term sepsis is derived from the greek word sepo, which means to putrefy or make putrid [1]. The early recognition, diagnosis, and management of sepsis remains one of the greatest challenges in the field of critical care medicine today. Part of this challenge historically has been the lack of an adequate, standardized definition of sepsis, prompting an international panel of experts from the Society of Critical Care Medicine (SCCM) and the American College of Chest Physicians (ACCP) to propose the now familiar consensus definitions for the systemic inflammatory response syndrome (SIRS), sepsis, severe sepsis, and septic shock in 1992 [2]. These definitions were reviewed and found to be acceptable again by an expert panel in 2001 [3]. These definitions have been modified for use in critically ill children with sepsis by the International Pediatric Sepsis Consensus Conference (IPSCC) [4] (see Tables 1 and $\mathbf{2}$ from the Introduction of this supplement). While far from perfect, these definitions have helped standardize the enrollment criteria for both observational and interventional trials for pediatric sepsis.

The systemic inflammatory response syndrome (SIRS) is therefore defined by the presence of at least two of the following four criteria: (1) Core temperature $>38.5 \mathrm{C}$ or $<36 \mathrm{C}$ (by rectal, bladder, oral or central catheter probe); (2) Tachycardia in the absence of pain, fever, drug therapy or bradycardia; (3) Tachypnea or need for mechanical ventilatory support; (4) Increased or decreased peripheral white blood cell (WBC) count. SIRS may result from infection or from a variety of sterile conditions, including

*Address correspondence to this author at the Department of Critical Care Medicine, Children's Hospital of Pittsburgh, Children's Hospital Drive, $45^{\text {th }}$ St. and Penn Ave, Pittsburgh, PA 15201, USA; Tel: (412) 692- 5164; Fax: (412) 692-6076; E-mail: anejar@upmc.edu pancreatitis, burns, trauma, and cardiopulmonary bypass. The term sepsis, on the other hand, refers specifically to a systemic inflammatory response to infection [4]. Infection is either suspected (on clinical grounds) or proven (i.e. by positive culture, tissue stain, or polymerase chain reaction). These criteria are relatively non-specific and may apply to as many as $82 \%$ (SIRS) and $23 \%$ (sepsis) of critically ill children admitted to the pediatric intensive care unit (PICU) [5]. Accordingly, some experts have argued that the lack of precision in the definitions of SIRS and hence, sepsis, renders them useless. Those who take this view suggest that patients should be classified more narrowly, using terms such as acute generalized peritonitis (with or without organ dysfunction), community-acquired pneumonia, and purpura fulminans associated with meningococcal infection. Regardless, however, we feel that the definitions for sepsis remains a valid and clinically useful concept that can be honed and refined over time, particularly if reliable biochemical markers can be added to the definition.

According to the consensus panel, severe sepsis is defined as sepsis plus one of the following: cardiovascular organ dysfunction, acute respiratory distress syndrome (ARDS), or two or more organ dysfunctions (respiratory, renal, neurologic, hematologic, or hepatic) (Table 1) [4]. Of note, simultaneous dysfunction of two or more organs systems has been defined as multiple organ failure [6]. Hence, it is important to remember that a child may have multiple organ failure with severe sepsis, i.e. without necessarily meeting the criteria for septic shock. Finally, septic shock is defined as sepsis in addition to cardiovascular organ dysfunction, manifested by fluid refractory hypotension, i.e. the need for an inotrope or vasopressor to maintain blood pressure, or two or more other indications of inadequate tissue perfusion (metabolic acidosis, elevated lactate, oliguria, and capillary refill $>5 \mathrm{sec}$ ) [4]. 
Table 1. Categories and Definitions of Organ Dysfunction in Pediatric Sepsis

\section{Cardiovascular}

- Hypotension ( $\mathrm{BP}<5$ th percentile for age or systolic $\mathrm{BP} 2 \mathrm{SD}$ below normal for age)

OR

- Requirement for vasoactive medication to maintain normal BP

OR

- Two of the following criteria:

Metabolic acidosis

Elevated lactate

Oliguria

Delayed capillary refill

Core-to-peripheral temperature gap

Respiratory

- $\mathrm{PaO} 2 / \mathrm{FiO} 2<300$ (not resulting from chronic lung disease or intracardiac shunting)

OR

- $\mathrm{PaCO} 2>65$ torr or $20 \mathrm{~mm} \mathrm{Hg}$ over baseline

OR

- Requirement for $\mathrm{FiO}_{2}>0.5$ to maintain oxygen saturation $\geq 92 \%$

OR

- Requirement for new mechanical ventilatory support

Neurologic

- Glasgow Coma Score (GCS) $\leq 11$

OR

- Acute decrease in GCS $\geq 3$ points from abnormal baseline

Hematologic

- Platelet count $<80,000 / \mathrm{mm} 3$ (or a drop of $50 \%$ from highest value over the previous three days)

OR

- International normalized ratio $>2$

Renal

- Serum creatinine $\geq$ twice normal for age or two-fold increase from baseline

Hepatic

- Total bilirubin $\geq 4 \mathrm{mg} / \mathrm{dL}$ (except in newborn)

OR

- ALT twice the upper limit of normal

The astute reader will note that the expert consensus panel's definition for shock [4] differs slightly from that definition used by the American College of Critical Care Medicine/American Heart Association's Pediatric Advanced Life Support (ACCM/PALS) guidelines [7, 8]. The ACCM/PALS guidelines define shock as sustained tachycardia, decreased peripheral pulses compared with central pulses, delayed capillary refill ( $>2$ secs, not $>5$ secs) or flash capillary refill, altered mental status, mottled cool extremities, or decreased urine output $(<1 \mathrm{~mL} / \mathrm{kg} / \mathrm{hr})$. Children possess a remarkable ability to maintain blood pressure through compensatory tachycardia and elevated systemic vascular resistance, and hypotension is therefore often a late sign of shock. While hypotension is not needed for the ACCM/PALS definition of septic shock, its presence is confirmatory of shock state in a child with suspected or proven infection [9].

So what definition should be used in pediatric septic shock? As both groups state in their discussion, each definition serves its purpose $[10,11]$. While the IPSCC definition was originally developed to facilitate standardized enrollment criteria for use in multicenter, randomized, controlled drug trials for children with "late-stage" septic shock, the ACCM/PALS guidelines target are helpful for the early recognition of shock and delivery of goal-directed therapy, as discussed later in this supplement. In practice, both definitions are quite useful and should therefore be easily recalled and used by anyone providing care to critically ill children.

\section{EPIDEMIOLOGY}

There are comparatively few high-quality epidemiologic studies of sepsis that have been conducted in children. In the largest and most comprehensive epidemiologic study on pediatric sepsis published to date, Watson and colleagues analyzed hospital discharge data (representing approximately one quarter of the U.S. population) and reported that sepsis accounted for nearly 4,500 deaths (mortality rate $10 \%$ ) and close to $\$ 2$ billion per year in healthcare expenditures in the U.S. alone in 1995 [12, 13]. The incidence of sepsis was highest in infants (5.16 cases per 1,000 population per year) and lowest in older children ( 0.2 cases per 1,000 population per year). Prematurity and the presence of pre-existing comorbidities were important risk factors for sepsis. Almost a third of the patients had respiratory infections as the inciting event and another 25\% were diagnosed with primary bacterial infections $[12,13]$.

Watson and colleagues reported the results of a follow-up study nearly a decade later and noted that while the annual incidence of pediatric sepsis has decreased from 0.56 per 1,000 population per year to 0.51 per 1,000 population per year, the total number of cases of pediatric sepsis has 


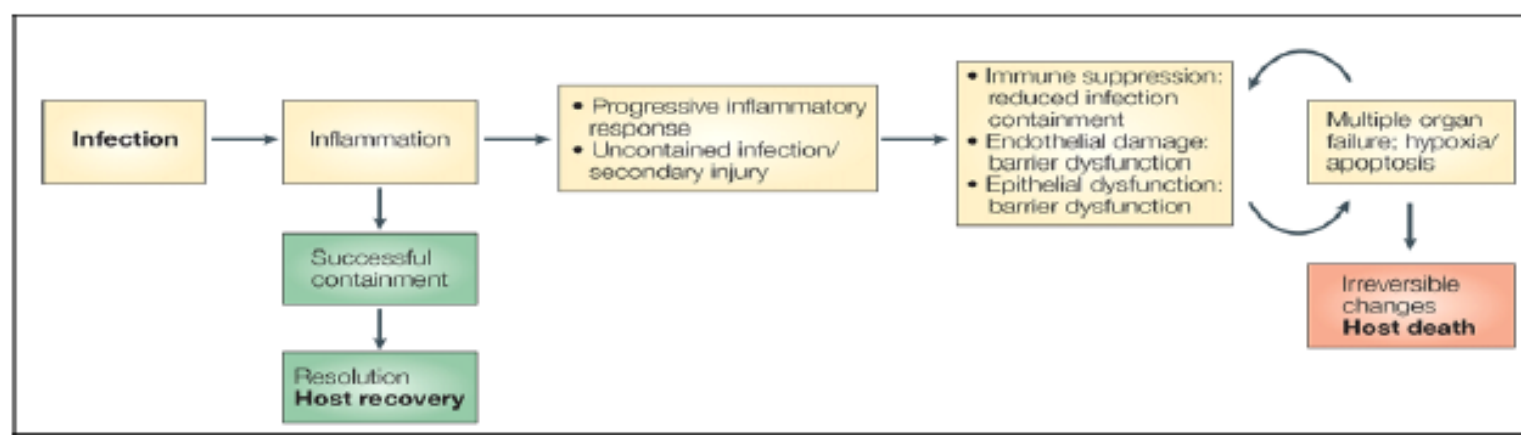

Fig. (1). The pathophysiology of sepsis resulting in multiple organ failure and death.

remained the same ( $\sim 42,000$ cases per year), due to an increase in the size of the pediatric population during this time. More concerning, the results of this follow-up study suggest that the incidence of sepsis appears to be dramatically increasing in children less than 12 months of age. Notably, while the overall incidence has decreased, the incidence of sepsis in this age group has increased by more than 50 percent. Further work is in progress to ascertain the cause for the dramatic increase in sepsis incidence in this age group. Somewhat more reassuring is the fact that the absolute mortality from sepsis has decreased from 10.3 to $7.6 \%$ in the last decade. Respiratory and blood infections continue to be the predominant causes of sepsis in the pediatric age group. However, compared to respiratory infections, the presence of bacteremia was a significant and independent risk factor for increased hospital length of stay and mortality. Consistent with previous reports, mortality was nearly $50 \%$ higher in critically ill children with preexisting conditions as compared to their healthy cohorts [14].

In a similar study, Odetola and colleagues examined the 2003 Kids' Inpatient Database (KID). KID was developed by the Agency for Healthcare Research and Quality (AHRQ) and includes nearly 3 million pediatric discharge records obtained from 3,438 hospitals in 36 states [15]. Almost a third of the hospitalized children were noted to have a preexisting condition. The overall mortality rate in children hospitalized with sepsis was $4.2 \%$. Collectively, these studies suggest that the overall mortality from pediatric sepsis appears to be improving, though the presence of preexisting, co-morbid conditions remains a significant risk factor for mortality [16].

\section{PATHOPHYSIOLOGY OF SEPSIS}

Sepsis is increasingly recognized as a systemically dysregulated inflammatory network that involves the host innate response, the host adaptive immune response, and activation of the complement system, coagulation cascades, and the neuro-endocrine systems (Fig. 1) [17, 18] . Many aspects of the pathophysiology of sepsis are covered in greater detail in subsequent reviews in this supplement. We will therefore focus on four key pathways - namely, endothelial activation, nitric oxide, activation of the coagulation cascade, and apoptosis.

\section{Endothelial Activation}

The vascular endothelium is a single layer of cells that lines the entire vascular bed, covering an estimated surface area of 4,000-7,000 $\mathrm{m}^{2}[19,20]$. The vascular endothelium was previously thought to be a relatively inert layer of cells that separated the blood and tissue. It is now widely understood that the vascular endothelium plays crucial roles in modulating the host immune response, regulating vascular growth and integrity, and regulating the levels of coagulation, pro-inflammatory, anti-inflammatory, and vasoactive factors in the blood [19, 21-23]. As such, the vascular endothelium may be considered the largest organ in the human body. The vascular endothelium is a semipermeable barrier that is particularly important in controlling the passage of macromolecules and fluid between the blood and interstitial space. Dysfunction of the vascular endothelium leads to capillary leak resulting in loss of fluid and/or protein from the vascular compartment into the interstitial compartment. Capillary leak is one of the sentinel signs of critical illness. The intravascular fluid depletion in capillary leak can further worsen hypotension and shock, thereby independently exacerbating tissue oxygen delivery. In pediatric septic shock, there is high incidence of capillary leak syndrome leading to relative hypovolemia and a decreased preload. Therefore, aggressive fluid resuscitation is recommended in the treatment of septic shock [24].

The inflammatory cascade may be activated by a diverse group of molecular motifs found on pathogens, known as pathogen-associated molecular patterns (PAMPs). These molecular motifs are in turn recognized by a surprisingly limited number of highly conserved pattern recognition receptors (PRRs), which include the Toll-like receptors (TLRs) and nucleotide-binding oligomerization domain (NOD) receptors. Importantly, these PRRs recognize both exogenous danger signals [25], which include PAMPs such as lipopolysaccharide (LPS), lipoteichoic acid, and peptidoglycan [26, 27], as well as endogenous danger signals [28], including uric acid [29] and extracellular heat shock proteins (HSPs) [30]. Perhaps the most well studied PAMP is LPS, a component of the outer membrane of gram negative bacteria. LPS, and in particular its lipid A component, is a key initiator of endothelial barrier dysfunction during sepsis. Exposure to LPS initiates endothelial dysfunction as early as $2 \mathrm{~h}$ following exposure, with progressively worsening endothelial dysfunction thereafter [31-35]. The morphological changes that characterize this response include cell contraction, disruption of interendothelial junctions, and loss of focal contacts with the underlying extracellular matrix [34]. LPS signals via the TLR-4/CD14 complex (see review on TLR in this 


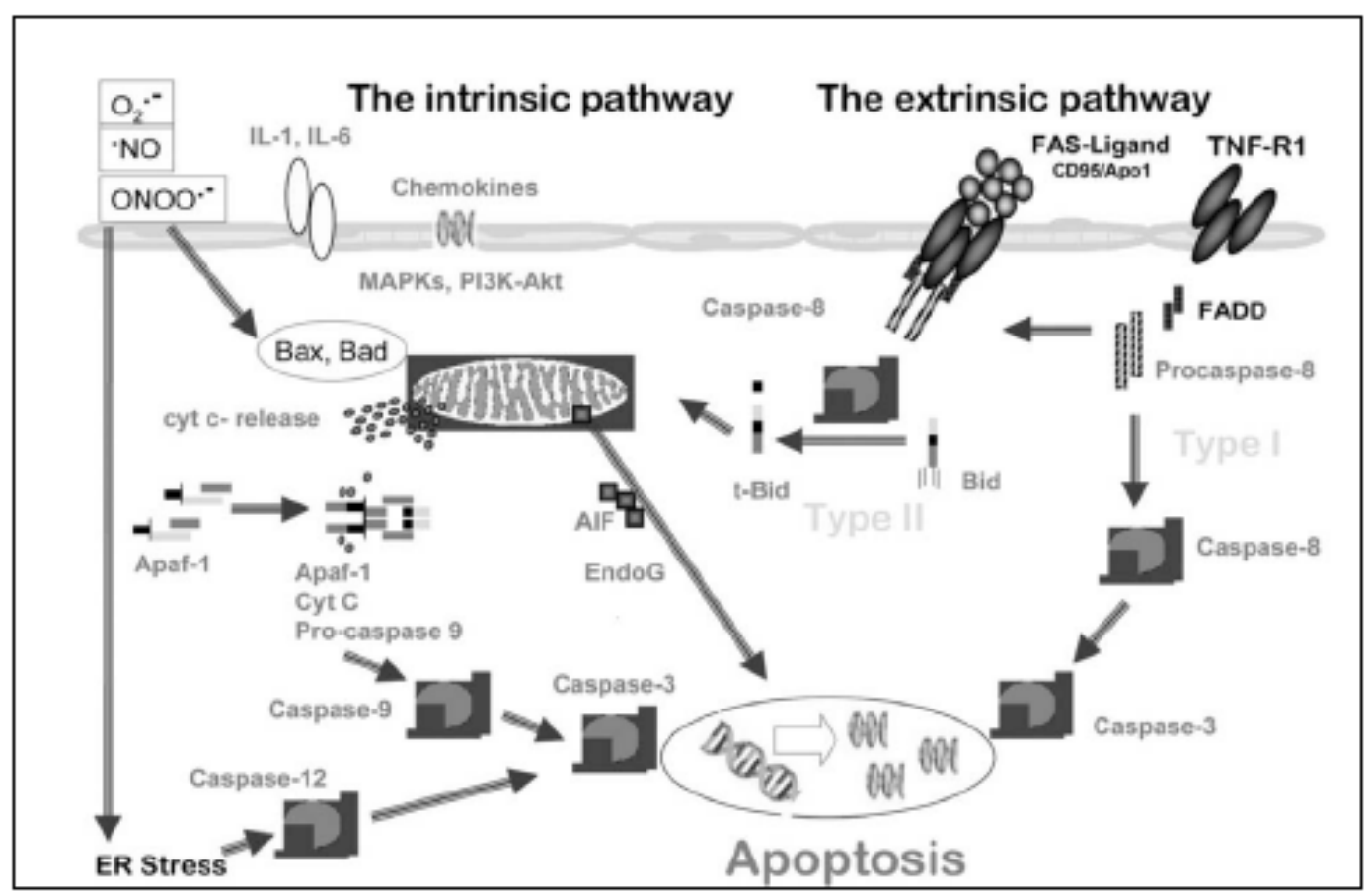

Fig. (2). The apoptotic signaling pathways. There are two main pathways involved in the induction of apoptosis; a death-receptor-initiated caspase-8-mediated pathway (known as the extrinsic pathway) and a mitochondria-initiated caspase-9-mediated pathway (known as the intrinsic pathway). (MAPK, mitogen-activated phosphokinase; PI3K/Akt, phosphoinositide3-kinase/Akt; $A P A F-1$, apoptosis protease activating factor $1 ; E R$, endoplasmic reticulum; $I L$, interleukin.).

supplement) and further downstream activates inhibitors of

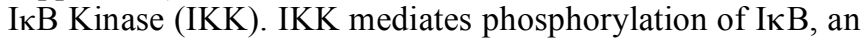
inhibitor of the transcription factor NF- $\mathrm{BB}$, which leads to I $\mathrm{B}$ degradation through the proteasome pathways and enables NF- $\kappa \mathrm{B}$ to translocate to the nucleus, resulting in proinflammatory cytokine gene expression.

Recently, High Mobility Group Box-1 (HMGB1) has been characterized as a late mediator of sepsis. HMGB1 is a DNA binding protein that belongs to an increasing number of endogenous PAMPs that are often collectively referred to as alarmins or danger-associated molecular patterns (DAMPs) [36, 37]. HMGB1 serves as a ligand for both TLR2 and TLR4, as well as the receptor for advanced glycation end-products (RAGE) [38-40]. HMGB1 is a late mediator of sepsis, and administration of HMGB1 antibody is protective in an LPS model of sepsis [38, 40-43]. Elevated levels of plasma HMGB1 have been demonstrated in critically ill patients with sepsis and septic shock. For example, critically ill children admitted with septic shock and multiple organ failure have significantly elevated levels of HMGB1 as compared to controls (Aneja, unpublished data). Furthermore, in contrast to the early response cytokines (IL-1 and TNF), HMGB1 peaks at 18-24 hours, making it a potential therapeutic target [43]. Further work is in progress to understand the importance of HMGB1 as an alarmin and its role in modulating the innate immune response.

In response to inflammation and tissue injury, neutrophils and other leukocytes transmigrate from the vascular compartment into the inflamed tissue space. The leukocytes roll along the vessel wall, followed by firm adhesion to the wall and migration across it. Gautam et al defined a paracrine mechanism in the cellular cross-talk between activated neutrophils and endothelial cells that regulate macromolecular efflux in conjunction with leukocyte trafficking in inflammation [44]. Adhesion molecules, including intercellular adhesion molecule (ICAM)-1 and vascular cell adhesion molecule (VCAM)-1, mediate firm adhesion between leukocytes and endothelium and subsequent diapedesis. Elevated plasma concentrations of ICAM-1, and VCAM-1 were increased in children with sepsis and children with more than three organ failures [45]. Neutrophils destroy pathogens through the release of proteolytic enzymes and reactive oxygen species and sequester them through the formation of neutrophil extracellular traps (NET), but may contribute to organ injury through collateral damage to the endothelium and activation of coagulation cascades [46, 47]. LPS can also induce endothelial apoptosis (discussed further below), an ATP dependent form of cell death, characterized by chromatin condensation, nuclear fragmentation, cell shrinkage and blebbing of the plasma membrane [48, 49].

\section{Nitric Oxide}

In 1984, Furchgott et al reported an endothelial derived relaxing factor that was subsequently found to be nitric oxide (NO) $[50,51]$. NO is a highly reactive nitrogen radical that is produced by three isoforms of the enzyme NO synthase. The two constitutive NO synthases are endothelial neuronal NO synthase (eNOS) and neuronal NO synthase (nNOS). The third NO synthase (inducible NO synthase [Inos]) is upregulated in response to inflammation [52, 53]. Nitric 
oxide (NO) synthase catalyzes the five-electron oxidation of L-arginine to NO, with the concomitant formation of Lcitrulline. The cofactors necessary for this reaction are oxygen and NADPH. Inducible NOS is expressed and activated in a variety of cells, including vascular endothelial cells, smooth muscle cells, macrophages and different parenchymal cells. NO activates the cyclic guanosine monophosphate (cGMP)-producing enzyme soluble guanylate cyclase (sGC), leading to activation of the cGMPdependent protein kinases (PKGs). The net effect of this cascade of events is reduction of cytosolic calcium levels and the sensitivity of the contractile apparatus to cytoplasmic calcium levels, ultimately leading to vasorelaxation. $\mathrm{NO}$ is a crucial mediator regulating the distribution of blood flow, ensuring adequate splanchnic and hepatic blood flow [54]. Excessive NO synthesis and release locally results in microvascular shunting (as in the hepatopulmonary and heptorenal synromes), while excessive systemic NO causes hypotension, splanchnic ischemia, increased gut mucosal permeability, and acute kidney injury [54-56]. NO may be an important circulating myocardial depressant factor and impairs mitochondrial function, leading to cellular dysoxia and organ injury [55, 57-59].

There is ample clinical evidence that NO plays a key role in sepsis. Hypotension in an experimental murine model of endotoxemia can be prevented by inhibitors of NO synthases [60], and significantly lower nitrite and nitrate levels have been found in animals treated with inhibitors of NO synthesis [61]. Plasma levels of NO metabolites, namely nitrate/nitrite, are elevated in children with septic shock and multi-organ failure [62, 63]. Generally, in adult patients, shock is characterized by low blood pressure and low systemic vascular resistance, along with upregulation of NOS enzymes. Accordingly, there has been significant enthusiasm for the potential of therapeutic inhibitors of NOS in the management of critically ill patients with septic shock. Unfortunately, a recently published randomized, placebocontrolled phase III trial with the NOS inhibitor, 546C88 was terminated prematurely due to increased mortality in patients randomized to the treatment group. It is possible that NOS inhibition in these patients might have led to a paradoxic worsening of their acute circulatory failure or perhaps, more specifically, myocardial dysfunction. Alternatively, it is possible that NOS inhibition might be beneficial in a select group of patients as compared to all patients with inflammation.

As reviewed previously, the hemodynamic presentation of septic shock in children is quite different compared to adults. Many children (approximately 50\%) in fluid refractory dopamine resistant septic shock manifest low cardiac output, normal blood pressure and high systemic vascular resistance. With this presentation, the mainstay of therapy is afterload reduction by using NO donors i.e. nitroprusside or nitroglycerin [64]. As such, the nitric oxide pathway continues to be a focus for translational research in sepsis, and it is widely believed that therapeutic agents targeting this pathway will continue to be developed and tested, both in pre-clinical models of septic shock and in clinical studies performed in the intensive care unit.

\section{Activation of Coagulation}

Microcirculatory fibrin deposition is believed to contribute to organ injury in sepsis and results from dysregulation of an inflammatory network comprising innate immune signaling, complement, the contact system (kallikrein/kinin system), and coagulation cascades [46, 65, 66]. Activation of the endothelium and the coagulation cascade by inflammatory mediators produces a thrombotic microangiopathy, resulting in either the syndrome of disseminated intravascular coagulation (DIC) or a relatively newly recognized syndrome similar to thrombotic thrombocytopenia (TTP), called thrombocytopeniaassociated multiple organ failure (TAMOF) [67-69]. DIC has been defined as the activation of intravascular coagulation and subsequent intravascular fibrin formation, which results in either secondary fibrinolysis or inhibition of fibrinolysis [70]. An important mediator of the coagulation pathway is tissue factor. Tissue factor is a $47-\mathrm{kDa}$ glycoprotein constitutively expressed in the lung and kidney, and its expression can be induced in both endothelial cells and monocytes. Endothelial disruption exposes tissue factor to circulating blood elements and activates the clotting cascade. In sepsis, pro-inflammatory cytokines stimulate tissue factor expression in circulating mononuclear cells, further leading to systemic activation of coagulation. Tissue factor forms a complex with factor VII. Upon activation, the complex activates both factors IX and X. The next step in the coagulation cascade is conversion of prothrombin to thrombin by activated factor $\mathrm{X}$. Thrombin catalyzes the conversion of fibrinogen to fibrin and eventually leads to clot formation.

Tissue plasminogen activator (tPA) and urokinase plasminogen activator (uPA) activate plasmin, which in turn degrades the fibrin clot. The levels of natural anticoagulants, such as protein $\mathrm{C}$, protein $\mathrm{S}$, antithrombin III and tissue factor pathway inhibitor, are decreased in patients with sepsis. In other words, during sepsis, the normal microvascular milieu is converted from an anticoagulant and profibrinolytic state, to a predominantly procoagulant and antifibrinolytic one. DIC is clinically diagnosed by the presence of thrombocytopenia, decreased factors $\mathrm{V}$ and $\mathrm{X}$, decreased fibrinogen and increased D-dimers.

The three major anticoagulant pathways that regulate activation of coagulation, namely antithrombin III, the protein C system, and tissue factor pathway inhibitor (TFPI), are dysfunctional in sepsis. We will mainly focus on activated protein $\mathrm{C}$ (APC). The conversion to APC is augmented by endothelial PC receptor (EPCR) which is present on endothelial cells, neutrophils and monocytes. The binding of thrombin to thrombomodulin results in an activation of protein $\mathrm{C}$ and also blocks the thrombinmediated conversion of fibrinogen into fibrin. Activated Protein $\mathrm{C}$ inactivates factors Va and VIIIa and plasminogen activator inhibitor (PAI)-1. Protein C activity is deficient in patients with sepsis. In an open-label phase 2 (Extended Evaluation of Recombinant Activated Protein C, or ENHANCE) trial in pediatric severe sepsis patients, $92 \%$ of the enrolled patients had protein C deficiency and $40 \%$ had severe protein $\mathrm{C}$ deficiency [71]. Possible causes for the protein $\mathrm{C}$ deficiency include impaired synthesis of protein $\mathrm{C}$ or downregulation of thrombomodulin on the endothelial 
surface [72]. Recombinant APC has been used successfully in several pre-clinical models of sepsis. Drotecogin alfa (activated [DrotAA]; Xigris ${ }^{\circledR}$; Eli Lilly and Company, Indianapolis, IN, USA) the recombinant form of the natural anticoagulant activated protein $\mathrm{C}$ was approved by the US Food and Drug Administration (FDA) in November 2001 for the treatment of adult patients with severe sepsis at high risk of death (for instance, as indicated by Acute Physiology and Chronic Health Evaluation [APACHE] II score). This was followed by a phase III randomized, double-blind, placebocontrolled trial (RESOLVE) evaluated the efficacy and safety of DrotAA ( $24 \mu \mathrm{g} / \mathrm{kg}$ per hour for 96 hours) in severe sepsis and included 477 pediatric patients (age range: $\geq 38$ weeks of gestation to $<18$ years)[73]. There was no significant difference between the two groups in the time to complete organ failure resolution, the study's primary endpoint. Mortality at 28 days was $17.5 \%$ in the placebo group and $17.2 \%$ in the DrotAA group $(\mathrm{P}=0.93)$. There were significant bleeding risks in the DrotAA groups, with increased episodes of intracranial hemorrhage in the DrotAA group (11 [4.6\%]) compared with in the placebo group (5 $[2.1 \%] ; \mathrm{P}=0.13)$. This difference was predominantly observed in children younger than 60 days at the time of enrollment. Hence the use of DrotAA is limited to young adults and currently is not being used in children admitted with sepsis and MOF.

Von Willebrand factor, a mediator of platelet adhesion and aggregation, is synthesized by the endothelial cells and is predominantly stored in specialized granules, called Weibel-Palade bodies. Under normal conditions, the ultra large and thrombogenic VWF multimers are cleaved by ADAMTS-13 (a disintegrin and metalloproteinase with thrombospondin-1-like domains) to smaller, less thrombogenic multimers [74]. ADAMTS-13 is synthesized in the liver and endothelium, and mechanisms for its reduced activity in sepsis are currently unknown. Acquired ADAMTS-13 deficiency leads to accumulation of ultra large VWF, leading to deposition of platelet thrombi in the microvasculature, especially in the brain and kidney. The presence of thrombocytopenia and multiple organ failure (organ failure index $\geq 3$ ) in children with pediatric sepsis has recently been called TAMOF. Since no rapid tests for ADAMTS-13 are currently available, the diagnosis for TAMOF is largely based upon clinical grounds. In a recent Scottish 2 year epidemiological surveillance study of thrombotic microangiopathies, almost $20 \%$ of patients presenting with TTP had severe sepsis [75]. Untreated TTP has a high mortality rate, but treatment with plasma exchange replenishes ADAMTS-13, removes ADAMTS-13 inhibitors, and removes thrombogenic ULVWF multimers [76]. Several investigators have suggested a similar approach to TAMOF secondary to sepsis. For example, preliminary studies at Children's Hospital of Pittsburgh demonstrate a reduction in mortality and resolution of organ failure with plasma exchange therapy in critically ill children with TAMOF $[67,76]$.

\section{Apoptosis}

Necrosis is a non-programmed form of cell death that is characterized by a condensed cellular appearance, swollen or ruptured mitochondria; disaggregation and detachment of ribosomes; disrupted organelle membranes; and swollen, ruptured lysosomes. This leads to eventual disruption of the cell membrane followed by release of cellular contents into the extracellular compartment. A different form of cellular death, called apoptosis (Greek: apo - from, ptosis - falling) is a homeostatic mechanism to maintain cell population in tissues and occurs normally during development and aging. It is characterized by cell shrinkage, condensation of the nuclear chromatin, activation of endonucleases and disruption of the mitochondrial transmembrane potential [77].

There are two main pathways involved in the induction of apoptosis; a death-receptor-initiated cysteine-dependent aspartate-specific proteases (caspase) -8-mediated pathway (known as the extrinsic pathway) and a mitochondrialinitiated caspase-9-mediated pathway (known as the intrinsic pathway) (Fig. 2). The extrinsic signaling pathway involves death receptors, that are members of the tumor necrosis factor gene superfamily [78, 79]. These protein receptors include. Fas (CD95 or Apo1), tumor necrosis factor receptor 1 (TNFR1 or p55), DR3 (TNF receptor apoptosis-mediating protein [TRAMP]), DR4 (Apo2 or TNF related apoptosisinducing ligand [TRAIL]-R1) and DR5 (TRAILR2). Upon binding of specific ligands to these receptors, cytoplasmic adapter proteins are recruited, leading to the formation of a death-inducing signaling complex (DISC). The formation of the DISC complex leads to the activation of the execution phase of apoptosis. In contrast to the extrinsic pathway, cells can activate the apoptosis pathway from inside in response to injury, hypoxia, starvation or loss of apoptotic suppression. Pro-apoptotic members like members of the B-cell $\mathrm{CCI} /$ lymphoma 2 (Bcl-2) family lead to loss of the mitochondrial membrane potential (Äøm), leading to the release of apoptogenic factors like cytochrome C. Cytosolic cytochrome $\mathrm{c}$ binds and activates Apoptotic peptidase activating factor (Apaf) -1 as well as procaspase-9, forming an "apoptosome" [80]. Other additional apoptosis inducers have been identified that can activate caspase 3 .

Both extrinsic and intrinsic pathways converge at the execution phase, also called the final pathway of apoptosis. In this phase, cytoplasmic endonuclease degrades nuclear material while the proteases degrade the nuclear and cytoskeletal proteins. Caspases also degrade various other substrates, cytokeratins, Poly (ADP)-ribose polymerase-1 leading to the characteristic morphology of apoptosis. The last phase, involves the externalization of phosphatidylserine on the surface of apoptotic cells and subsequent uptake by the phagocytes.

Apoptosis causes immune suppression by two mechanisms. Apoptosis induces depletion of the T and B cells, impairing the adaptive immune response and subsequently impairing the innate immune response. Furthermore, macrophages or dendritic cells (DCs) that ingest apoptotic cells shift to a T helper 2 (TH2) phenotype or become anergic [81]. Similarly, other studies have also documented apoptotic depletion of cells of the innate and adaptive immune system [82, 83]. This leads to immune suppression, impairing the host's immune defenses. Felmet et al enrolled 58 critically ill children with and 55 without MOF in a prospective, longitudinal, observational clinical study. Out of the 16 patients that died in this study, only 11 underwent autopsy. Most of the children (89\%) who died 
with MOF were noted to have severe lymphocyte depletion. The one patient who died with MOF without lymphocyte depletion had a rapid course with fulminant hepatic failure. Patients who died without MOF did not show any evidence of lymphocyte depletion [84] . It is important to point out that almost all patients who died of MOF with lymphoid depletion had autopsy evidence of unresolved nosocomial infection.

\section{CONCLUSION}

Since the term "sepsis" was first coined three thousand years ago, we have come a long way in our understanding of the pathophysiology of sepsis. Despite our advancement, the only therapy that has been translated successfully from the laboratory to the bedside is activated protein C. This therapy has been approved for adults, but not for children. Hence, we still have a long way to go before we can overcome the limitations in our knowledge of molecular mechanisms in sepsis and design innovative therapies for pediatric sepsis.

\section{REFERENCES}

[1] Geroulanos S, Douka ET. Historical perspective of the word "sepsis". Intense Care Med 2006; 32: 2077.

[2] Bone RC, Balk RA, Cerra FB, et al. Definitions for sepsis and organ failure and guidelines for the use of innovative therapies in sepsis. The ACCP/SCCM Consensus Conference Committee. American College of Chest Physicians/Society of Critical Care Medicine. Chest 1992; 101(6): 1644-55.

[3] Levy MM, Fink MP, Marshall JC, et al. 2001 SCCM/ESICM/ACCP/ATS/SIS International Sepsis Definitions Conference. Crit Care Med 2003; 31: 1250-6.

[4] Goldstein B, Giroir B, Randolph A. International pediatric sepsis consensus conference: definitions for sepsis and organ dysfunction in pediatrics. Pediatr Crit Care Med 2005; 6: 2-8.

[5] Proulx F, Fayon M, Farrell CA. Epidemiology of sepsis and multiple organ dysfunction syndrome in children. Chest 1996; 109: 1033-7.

[6] Wilkinson JD, Pollack MM, Glass NL, et al. Mortality associated with multiple organ system failure and sepsis in pediatric intensive care unit. J Pediatr 1987; 111: 324-8.

[7] Oliveira CF, Noqueira de Sa FR, Oliveira DS, et al. Time- and fluid-sensitive resuscitation for hemodynamic support of children in septic shock: Barriers to the implementation of the American College of Critical Care Medicine/Pediatric Advanced Life Support Guidelines in a pediatric intensive care unit in a developing world. Pediatr Emerg Care 2008; 24: 810-5.

[8] de Oliveira CF, de Oliveira DS, Gottschald AF, et al. ACCM/PALS haemodynamic support guidelines for paediatric septic shock: An outcomes comparison with and without monitoring central venous oxygen saturation. Intensive Care Med 2008; 34: 1065-75.

[9] Carcillo JA, Fields AI. Clinical practice parameters for hemodynamic support of pediatric and neonatal patients in septic shock. Crit Care Med 2002; 30: 1365-78.

[10] Goldstein B, Giroir B, Randolph A. Reply: definitions of pediatric septic shock. Pediatr Crit Care Med 2005; 6: 501

[11] Kuch BA, Carcillo JA, Han YY, Orr RA. Definitions of pediatric septic shock. Pediatr Crit Care Med 2005; 6: 501.

[12] Watson RS, Carcillo JA. Scope and epidemiology of pediatric sepsis. Pediatr Crit Care Med 2005; 6: S3-5.

[13] Watson RS, Carcillo JA, Linde-Zwirble WT, et al. The epidemiology of severe sepsis in children in the United States. Am J Respir Crit Care Med 2003; 167: 695-701.

[14] Hartman M, Clermont G, Angus D, Watson R. Pediatric Severe Sepsis in the US: 1995 vs. 2005. Crit Care Med 2008; 36, : A76.

[15] Agency for Healthcare Research and Quality. Healthcare Cost and Utilization Project (HCUP) Kids' Inpatient Database (KID) 2003: Description of Data Elements. Rockville, MD. Agency for Healthcare Research and Quality; 2005.

[16] Odetola FO, Gebremariam A, Freed GL. Patient and hospital correlates of clinical outcomes and resource utilization in severe pediatric sepsis. Pediatrics 2007; 119: 487-94.
[17] Rittirsch D, Flierl MA, Nadeau BA, et al. Functional roles for C5a receptors in sepsis. Nat Med 2008 ; 14: 551-7.

[18] Buras JA, Holzmann B, Sitkovsky M. Animal models of sepsis: setting the stage. Nat Rev Drug Discov 2005; 4: 854-65.

[19] Aird WC. The role of the endothelium in severe sepsis and multiple organ dysfunction syndrome. Blood 2003; 101: 3765-77.

[20] Aird W. Endothelium as an organ system. Crit Care Med 2004; 32: S271-S9.

[21] Wolinsky H. A proposal linking clearance of circulating lipoproteins to tissue metabolic activity as a basis for understanding atherogenesis. Circ Res 1980; 47: 301-11.

[22] Cines DB, Pollak ES, Buck CA, et al. Endothelial cells in physiology and in the pathophysiology of vascular disorders. Blood 1998; 91: 3527-61.

[23] Gross PL, Aird WC. The endothelium and thrombosis. Semin Thromb Hemost 2000; 26: 463-78.

[24] Carcillo JA, Davis AL, Zaritsky A. Role of early fluid resuscitation in pediatric septic shock. JAMA 1991; 266: 1242-5.

[25] Matzinger P. An innate sense of danger. Semin Immunol 1998; 10: 399-415.

[26] Akira S, Takeda K. Toll-like receptor signaling. Nat Rev Immunol 2004; 4: 499-511.

[27] Franchi L, McDonald C, Kanneganti T-D, Amer A, Nunez G. Nucleotide-binding oligomerization domain-like receptor $\mathrm{s}$ : Intracellular pattern recognition molecules for pathogen detection and host defense. J Immunol 2006; 177: 3507-13.

[28] Zhang X, Mosser DM. Macrophage activation by endogenous danger signals. J Pathol 2008; 214: 161-78.

[29] Shi Y, Evans JE, Rock KL. Molecular identification of a danger signal that alerts the immune system to dying cells. Nature 2003; 425: 516-21.

[30] El Mezayen R, El Gazzar M, Seeds MC, et al. Endogenous signals released from necrotic cells augment inflammatory responses to bacterial endotoxin. Immunol Lett 2007; 111:36-44.

[31] Goldblum SE, Brann TW, Ding X, Pugin J, Tobias PS. Lipopolysaccharide (LPS)-binding protein and soluble CD14 function as accessory molecules for LPS-induced changes in endothelial barrier function, in vitro. J Clin Invest 1994; 93: 692702 .

[32] Bannerman DD, Sathyamoorthy M, Goldblum SE. Bacterial lipopolysaccharide disrupts endothelial monolayer integrity and survival signaling events through caspase cleavage of adherens junction proteins. J Biol Chem 1998; 273: 35371-80.

[33] Meyrick BO, Ryan US, Brigham KL. Direct effects of E coli endotoxin on structure and permeability of pulmonary endothelial monolayers and the endothelial layer of intimal explants. Am J Pathol 1986; 122: 140-51.

[34] Bannerman DD, Goldblum SE. Direct effects of endotoxin on the endothelium: barrier function and injury. Laboratory investigation. J Tech Methods Pathol. 1999; 79: 1181-99.

[35] Goldblum SE, Ding X, Campbell-Washington J. TNF-alpha induces endothelial cell F-actin depolymerization, new actin synthesis, and barrier dysfunction. Am J Physiol Cell Physiol 1993; 264: C894-905.

[36] Wheeler DS, Wong HR. Heat shock response and acute lung injury. Free Radic Biol Med 2007; 42: 1-14.

[37] Klune JR, Dhupar R, Cardinal J, Billiar TR, Tsung A. HMGB1: Endogenous danger signaling. Mol Med 2008; 14: 476-84.

[38] Lotze MT, Tracey KJ. High-mobility group box 1 protein (HMGB1): nuclear weapon in the immune arsenal. Nat Rev Immunol 2005: 5: 331-42.

[39] Hori O, Brett J, Slattery T, et al. The receptor for advanced glycation end products (RAGE) is a cellular binding site for amphoterin. Mediation of neurite outgrowth and co-expression of rage and amphoterin in the developing nervous system. J Biol Chem 1995; 270: 25752-61.

[40] Park JS, Svetkauskaite D, He Q, et al. Involvement of toll-like receptors 2 and 4 in cellular activation by high mobility group box 1 protein. J Biol Chem 2004; 279: 7370-7.

[41] Wang H, Liao H, Ochani M, et al. Cholinergic agonists inhibit HMGB1 release and improve survival in experimental sepsis. Nat Med 2004; 10: 1216-21.

[42] Yang H, Ochani M, Li J, et al. Reversing established sepsis with antagonists of endogenous high-mobility group box 1. Proc Natl Acad Sci USA 2004; 101: 296-301. 
[43] Wang H, Yang H, Czura CJ, Sama AE, Tracey KJ. HMGB1 as a late mediator of lethal systemic inflammation. Am J Respir Crit Care Med 2001; 164: 1768-73.

[44] Gautam N, Olofsson AM, Herwald H, et al. Heparin-binding protein (HBP/CAP37): a missing link in neutrophil-evoked alteration of vascular permeability. Nat Med 2001; 7: 1123-7.

[45] Whalen MJ, Doughty LA, Carlos TM, et al. Intercellular adhesion molecule-1 and vascular cell adhesion molecule-1 are increased in the plasma of children with sepsis-induced multiple organ failure. Crit Care Med 2000; 28: 2600-7.

[46] Cohen J. The immunopathogenesis of sepsis. Nature 2002; 420: 885-91.

[47] Clark SR, Ma AC, Tavener SA, et al. Platelet TLR4 activates neutrophil extracellular traps to ensnare bacteria in septic blood. Nat Med 2007; 13: 463-9.

[48] Bannerman DD, Goldblum SE. Mechanisms of bacterial lipopolysaccharide-induced endothelial apoptosis. Am J Physiol Lung Cell Mol Physiol 2003; 284: L899-914.

[49] Hull C, McLean G, Wong F, Duriez PJ, Karsan A. Lipopolysaccharide signals an endothelial apoptosis pathway through TNF receptor-associated factor 6-mediated activation of cJun NH2-terminal kinase. J Immunol 2002; 169: 2611-8.

[50] Furchgott RF, Zawadzki JV. The obligatory role of endothelial cells in the relaxation of arterial smooth muscle by acetylcholine. Nature 1980; 288: 373-6.

[51] Palmer RM, Ferrige AG, Moncada S. Nitric oxide release accounts for the biological activity of endothelium-derived relaxing factor. Nature 1987; 327: 524-6.

[52] Cauwels A. Nitric oxide in shock. Kidney Int 2007; 72: 557-65.

[53] Fleming I, Busse R. Molecular mechanisms involved in the regulation of the endothelial nitric oxide synthase. Am J Physiol Regul 2003; 284: R1-12.

[54] Shah V, Lyford G, Gores G, Farrugia G. Nitric oxide in gastrointestinal health and disease. Gastroenterology 2004; 126: 903-13.

[55] Abraham E, Singer M. Mechanisms of sepsis-induced organ dysfunction. Crit Care Med 2007; 35: 2408-16.

[56] Schrier RW, Wang W. Acute renal failure and sepsis. N Engl J Med 2004; 351:159-69.

[57] Brealey D, Brand M, Hargreaves I, et al. Association between mitochondrial dysfunction and severity and outcome of septic shock. Lancet 2002; 360: 219-23.

[58] Cohen RI, Wilson D, Liu SF. Nitric oxide modifies the sarcoplasmic reticular calcium release channel in endotoxemia by both guanosine-3',5' (cyclic) phosphate-dependent and independent pathways. Crit Care Med 2006; 34: 173-81.

[59] Hill BG, Dranka BP, Bailey S, Lancaster J, Darley-Usmar V. What part of NO don't you understand? Some answers to the cardinal questions in nitric oxide biology. J Biol Chem 2010; 285(26): 19699-704.

[60] Nava E, Palmer RM, Moncada S. The role of nitric oxide in endotoxic shock: effects of NG-monomethyl-L-arginine. J Cardiovasc Pharmacol 1992; 20 (Suppl 12): S132-4.

[61] Tracey WR, Nakane M, Kuk J, et al. The nitric oxide synthase inhibitor, L-NG-monomethylarginine, reduces carrageenan-induced pleurisy in the rat. J Pharmacol Exp Ther 1995; 273: 1295-9.

[62] Doughty L, Carcillo JA, Kaplan S, Janosky J. Plasma nitrite and nitrate concentrations and multiple organ failure in pediatric sepsis. Crit Care Med 1998; 26: 157-62.

[63] Krafte-Jacobs B, Brilli R, Szabo C, et al. Circulating methemoglobin and nitrite/nitrate concentrations as indicators of nitric oxide overproduction in critically ill children with septic shock. Crit Care Med 1997; 25: 1588-93.
[64] Brierley J, Carcillo JA, Choong K, et al. Clinical practice parameters for hemodynamic support of pediatric and neonatal septic shock: 2007 update from the American College of Critical Care Medicine. Crit Care Med 2009; 37: 666-88.

[65] Rittirsch D, Flierl MA, Ward PA. Harmful molecular mechanisms in sepsis. Nat Rev Immunol 2008; 8: 776-87.

[66] Oehmcke S, Herwald H. Contact system activation in severe infectious diseases. J Mol Med 2010; 88: 121-6.

[67] Nguyen T, Carcillo J. Bench-to-bedside review: Thrombocytopeniaassociated multiple organ failure - a newly appreciated syndrome in the critically ill. Crit Care 2006; 10: 235.

[68] Kwan HC. Thrombotic microangiopathy. Semin Hematol 1987; 24 : 69-81.

[69] Kwan HC. Miscellaneous secondary thrombotic microangiopathy. Semin Hematol 1987; 24: 141-7.

[70] Müller-Berghaus G, Madlener K, Blombäck M, et al. DIC: Pathogenesis, diagnosis and therapy of disseminated intravascular fibrin formation. Amsterdam: Elsevier Science Publishers BV 1993.

[71] Goldstein B, Nadel S, Peters M, et al. ENHANCE: results of a global open-label trial of drotrecogin alfa (activated) in children with severe sepsis. Pediatr Crit Care Med 2006; 7: 200-11.

[72] Levi M. The coagulant response in sepsis. Clin Chest Med 2008; 29: 627-42, viii.

[73] Nadel S, Goldstein B, Williams MD, et al. Drotrecogin alfa (activated) in children with severe sepsis: a multicentre phase III randomised controlled trial. Lancet 2007; 369: 836-43.

[74] Dong JF, Moake JL, Nolasco L, et al. ADAMTS-13 rapidly cleaves newly secreted ultralarge von Willebrand factor multimers on the endothelial surface under flowing conditions. Blood 2002; 100: 4033-9.

[75] Pollock KG, Young D, Beattie TJ, Todd WT. Clinical surveillance of thrombotic microangiopathies in Scotland, 2003-2005. Epidemiol Infect 2008; 136: 115-21.

[76] Nguyen TC, Han YY, Kiss JE, et al. Intensive plasma exchange increases a disintegrin and metalloprotease with thrombospondin motifs-13 activity and reverses organ dysfunction in children with thrombocytopenia-associated multiple organ failure. Crit Care Med 2008; 36: 2878-87.

[77] Ayala A, Perl M, Venet F, et al. Apoptosis in sepsis: mechanisms, clinical impact and potential therapeutic targets. Curr Pharm Des 2008; 14: 1853-9.

[78] Locksley RM, Killeen N, Lenardo MJ. The TNF and TNF Receptor Superfamilies: integrating mammalian. Biol Cell 2001; 104: 487501.

[79] Cinel I, Opal SM. Molecular biology of inflammation and sepsis: a primer. Crit Care Med 2009; 37: 291-304.

[80] Chinnaiyan AM. The apoptosome: heart and soul of the cell death machine. Neoplasia 1999; 1: 5-15.

[81] Hotchkiss RS, Nicholson DW. Apoptosis and caspases regulate death and inflammation in sepsis. Nat Rev Immunol 2006; 6: 81322.

[82] Hotchkiss RS, Swanson PE, Freeman BD, et al. Apoptotic cell death in patients with sepsis, shock, and multiple organ dysfunction. Crit Care Med 1999; 27: 1230-51.

[83] Hotchkiss RS, Tinsley KW, Swanson PE, et al. Depletion of dendritic cells, but not macrophages, in patients with sepsis. J Immunol 2002; 168: 2493-500.

[84] Felmet KA, Hall MW, Clark RSB, Jaffe R, Carcillo JA. Prolonged lymphopenia, lymphoid depletion, and hypoprolactinemia in children with nosocomial sepsis and multiple organ failure. J Immunol 2005; 174: 3765-72. 